\title{
Comportamiento de la rigidez arterial en diferentes condiciones clínicas utilizando el método oscilométrico
}

\author{
Behavior of arterial stiffness in different \\ clinical conditions using the oscillometric method
}

\author{
Alejandro Pizano, Darío Echeverri, Jaime Cabrales, Félix R. Montes • \\ BOGOTÁ, D.C. (COLOMBIA) \\ Pedro Forcada • Buenos Aires (Argentina)
}

\section{Resumen}

El estudio de la rigidez arterial, en los últimos años ha sido de gran interés en la comunidad científica, siendo un indicador importante de riesgo cardiovascular y de mortalidad, además es un predictor de progresión para ciertas enfermedades crónicas. Con el fin de comprender la interpretación de la medición de la rigidez vascular, se describen 12 casos de pacientes con diferentes condiciones fisiopatológicas, que reflejan la práctica clínica diaria, en los que se evaluó la rigidez arterial mediante un método oscilométrico (Arteriograph ${ }^{\circledR}$, TensioMed, Budapest-Hungría, Ltd.), calculando la velocidad de onda de pulso, presiones arteriales periféricas y centrales, presión de pulso, e índices de aumentación. La medición e interpretación adecuada de esta información puede ayudar a entender y resolver dudas frente a diferentes condiciones fisiopatológicas asociadas a las enfermedades cardiovasculares, mejorando la toma de decisiones terapéuticas, el seguimiento de pacientes y optimizar la prevención cardiovascular. (Acta Med Colomb 2018; 43: 165-170).

Palabras clave: rigidez arterial; presión de pulso; velocidad de onda de pulso; enfermedades cardiovasculares.

\section{Abstract}

The study of arterial stiffness has been in recent years of great interest in the scientific community, being an important indicator of cardiovascular risk and mortality, in addition to being a predictor of progression for certain chronic diseases. In order to understand the interpretation of the measurement of vascular rigidity, 12 cases of patients with different physiopathological conditions are described, thus reflecting the daily clinical practice, in which the arterial stiffness was evaluated by means of an oscillometric method (Arteriograph ${ }^{\circledR}$, TensioMed , Budapest-Hungary, Ltd.), calculating the pulse wave velocity, peripheral and central arterial pressures, pulse pressure, and augmentation indices. The adequate measurement and interpretation of this information can help to understand and resolve doubts in the face of different physiopathological conditions associated with cardiovascular diseases, improving therapeutic decision-making, following-up of patients and optimizing cardiovascular prevention. (Acta Med Colomb 2018; 43: 165-170).

Keywords: arterial stiffness; pulse pressure; pulse wave velocity; cardiovascular diseases.
Dr. Alejandro Pizano: Laboratorio de Investigación en Función Vascular; Dr. Darío Echeverri: Laboratorio de Investigación en Función Vascular, Servicio de Hemodinamia; Dr. Jaime Cabrales: Servicio de Hemodinamia; Dr. Félix R. Montes: Laboratorio de Investigación en Función Vascular. Fundación CardioInfantil - Instituto de Cardiología, Bogotá, D.C., (Colombia).

Dr. Pedro Forcada: Laboratorio Vascular no-invasivo, Hospital Universitario Austral, Buenos Aires (Argentina).

Correspondencia: Dr. Alejandro Pizano Umaña, Bogotá, D.C. (Colombia).

Email: apizanou@gmail.com

Recibido: 15/XII/2017 Aceptado: 25/VI/2018

\section{Introducción}

La rigidez arterial es un proceso fisiológico relacionado al envejecimiento y deterioro vascular metabólico, considerada indicador independiente de morbilidad cardiovascular y mortalidad en general (1-4). Asimismo, es predictor de progresión de enfermedades; diabetes mellitus (5-6), hipertensión arterial (7-8), enfermedad renal crónica (9) y dislipidemia (10). Igualmente, se ha detectado incremento de la rigidez en pacientes con intolerancia a la glucosa, síndrome metabólico e hipertensión arterial, sugiriendo un valor predictivo (10-12).

Según la American Heart Association (AHA) (2), la velocidad de onda de pulso (VOP) es el patrón de referencia 
para estimar la rigidez arterial en pacientes mayores y en jóvenes los índices de aumentación arterial (Aix), según el Anglo-Cardiff Collaborative Trial (ACCT) (13). Existen diferentes métodos para medir la VOP. El patrón de referencia es invasivo, costoso y requiere radiación, por lo cual se han creado métodos no-invasivos, siendo el Arteriograph ${ }^{\circledR}$ (TensioMed Budapest Hungría, Ltd.) un método oscilométrico sencillo, validado y reproducible (2).

De esta manera, en los últimos años se ha generado interés en estudiar la rigidez vascular, especialmente la VOP, definida como la velocidad de la propagación de la onda de pulso arterial medida en $\mathrm{m} / \mathrm{seg}$ (1-2), y los índices de aumentación (Aix-braquial/central), definidos como el aumento de presión (AP) dividido por la presión de pulso (PP) expresados en porcentaje. El AP es la diferencia entre el primer y segundo pico de la onda, y PP es la diferencia entre la presión sistólica (PAS) y presión diastólica (PAD) (14-15).

La rigidez arterial central tiene importantes repercusiones hemodinámicas; aumento de la amplitud de presión, disminución de la tensión-tangencial (shear-stress), e incremento en la transmisión del flujo-pulsátil de la microcirculación (16). Se ha demostrado que una VOP elevada se asocia con enfermedad coronaria y accidente cerebrovascular (11). También, se han identificado procesos mecánicos que aumentan la VOP, como es el caso de la corrección de aneurismas aórticos con endoprótesis (17-18).

En el ambiente de nuestra práctica clínica, no es infrecuente que surjan preguntas: ¿Por qué medir la rigidez arterial? y ¿qué impacto tiene? La rigidez arterial es un parámetro que hoy en día es fácil de medir (dispositivos

Tabla 1. Presentación de casos; resumen historia clínica y parámetros vasculares.

\begin{tabular}{|c|c|c|c|c|c|c|c|c|}
\hline Caso & Características & Condición & Medicamentos & PAS/PAD & SBP & VOP & Edad & $\begin{array}{l}\text { Aix-B } \\
\text { Aix-C }\end{array}$ \\
\hline 1 & $\begin{array}{l}\text { Hombre de } 57 \text { años } \\
\text { sano }\end{array}$ & Sedentario & No & $110 / 72$ & 109 & 7.8 & 40 & $\begin{array}{l}-12 \\
32\end{array}$ \\
\hline 2 & $\begin{array}{l}\text { Hombre de } 57 \text { años } \\
\text { sano }\end{array}$ & Atleta* & No & $117 / 72$ & 108 & 7.2 & 33 & $\begin{array}{c}-40 \\
17\end{array}$ \\
\hline 3 & $\begin{array}{l}\text { Hombre de } 56 \text { años } \\
\text { asintomático }\end{array}$ & $\begin{array}{c}\text { Riesgo CV } \\
\text { moderado }\end{array}$ & No & $116 / 80$ & 109 & 9.9 & $>60$ & $\begin{array}{c}-43 \\
5.9\end{array}$ \\
\hline 4 & $\begin{array}{l}\text { Mujer de } 68 \text { años con } \\
\text { DA tipo B }\end{array}$ & $\begin{array}{c}\text { Endoprótesis, } \\
\text { HTA }\end{array}$ & $\begin{array}{l}\text { Metoprolol y } \\
\text { amlodipino }\end{array}$ & $125 / 80$ & 131 & 17.8 & $>60$ & $\begin{array}{l}79 \\
78\end{array}$ \\
\hline 5 & $\begin{array}{l}\text { Niña de } 17 \text { años con } \\
\text { HHF }\end{array}$ & TH, EAC y EA & $\begin{array}{l}\text { Aspirina y } \\
\text { clopidogrel }\end{array}$ & $120 / 62$ & 117 & 9.1 & $>60$ & $\begin{array}{c}-16 \\
30\end{array}$ \\
\hline 6 & $\begin{array}{l}\text { Hombre de } 53 \text { años } \\
\text { asintomático }\end{array}$ & HCL, LDL: 202 & No & $136 / 60$ & 140 & 9.7 & $>60$ & $\begin{array}{c}-2.2 \\
37\end{array}$ \\
\hline 7 & $\begin{array}{l}\text { Hombre de } 62 \text { años } \\
\text { asintomático }\end{array}$ & $\begin{array}{c}\text { HTA, EC } \\
\text { e HCL controladas }\end{array}$ & $\begin{array}{c}\text { Aspirina, } \\
\text { metoprolol, } \\
\text { enalapril, } \\
\text { rosuvastatina } \\
\text { y clopidogrel }\end{array}$ & 13987 & 129 & 7.4 & 36 & $\begin{array}{l}-2.7 \\
29\end{array}$ \\
\hline 8 & $\begin{array}{l}\text { Mujer de } 81 \text { años } \\
\text { asintomática }\end{array}$ & $\begin{array}{l}\text { HTA, SAHOS, } \\
\text { Aorta en } \\
\text { Porcelana }\end{array}$ & $\begin{array}{l}\text { Carvedilol, } \\
\text { aspirina y } \\
\text { atorvastatina }\end{array}$ & $160 / 66$ & 178 & 12.9 & $>60$ & $\begin{array}{l}49 \\
63\end{array}$ \\
\hline 9 & $\begin{array}{l}\text { Mujer de } 59 \text { años } \\
\text { asintomática }\end{array}$ & $\begin{array}{l}\text { HCL, EC, HTA, No } \\
\text { controladas }\end{array}$ & $\begin{array}{l}\text { Losartan y } \\
\text { metoprolol }\end{array}$ & $152 / 87$ & 151 & 10.1 & $>60$ & $\begin{array}{c}-11 \\
32\end{array}$ \\
\hline 10 & $\begin{array}{l}\text { Hombre de } 26 \text { años } \\
\text { asintomático }\end{array}$ & $\begin{array}{l}\text { HTA } \\
\text { Resistente } \\
\text { desde hace } \\
9 \text { años }\end{array}$ & $\begin{array}{l}\text { Ibabradina, } \\
\text { telmisartan, } \\
\text { clonidina, } \\
\text { alfametildopa y } \\
\text { minoxidil }\end{array}$ & $162 / 97$ & 140 & 8.6 & 50 & $\begin{array}{l}-84 \\
-4.8\end{array}$ \\
\hline 11 & $\begin{array}{l}\text { Niña de } 12 \text { años } \\
\text { asintomática }\end{array}$ & CA sin corrección & Metoprolol & $195 / 128$ & 205 & 11.1 & $>60$ & $\begin{array}{l}19 \\
47\end{array}$ \\
\hline 12 & $\begin{array}{l}\text { Niño de } 12 \text { años } \\
\text { asintomático }\end{array}$ & $\begin{array}{l}\text { CA y corrección } \\
\text { con stent }\end{array}$ & Metoprolol y aspirina & $149 / 76$ & 136 & 6.4 & 18 & $\begin{array}{c}-39 \\
18\end{array}$ \\
\hline \multicolumn{9}{|c|}{$\begin{array}{l}\text { PAS: Presión Arterial Sistólica (mmHg), PAD: Presión Arterial Diastólica Braquial (mmHg), SBP: Presión Sistólica Central (mmHg), VOP: Velocidad de Onda de Pulso (m/seg), } \\
\text { Edad: vascular (años), Aix: Índice de Aumentación (\%), B: Braquial, C: Central. DA: Disección de Aorta; SAHOS: Síndrome de Apnea Obstructiva del Sueño; } \\
\text { HHF: Hipercolesterolemia Homocigota Familiar; CV: Cardiovascular; HTA: Hipertensión arterial; TH: Trasplante Hepático; EAC: Enfermedad Arterial Coronaria; } \\
\text { EA: Estenosis Aortica; HCL: Hipercolesterolemia; CA: Coartación de Aorta. * Ejercicio aeróbico por una hora más de tres veces por semana. }\end{array}$} \\
\hline
\end{tabular}




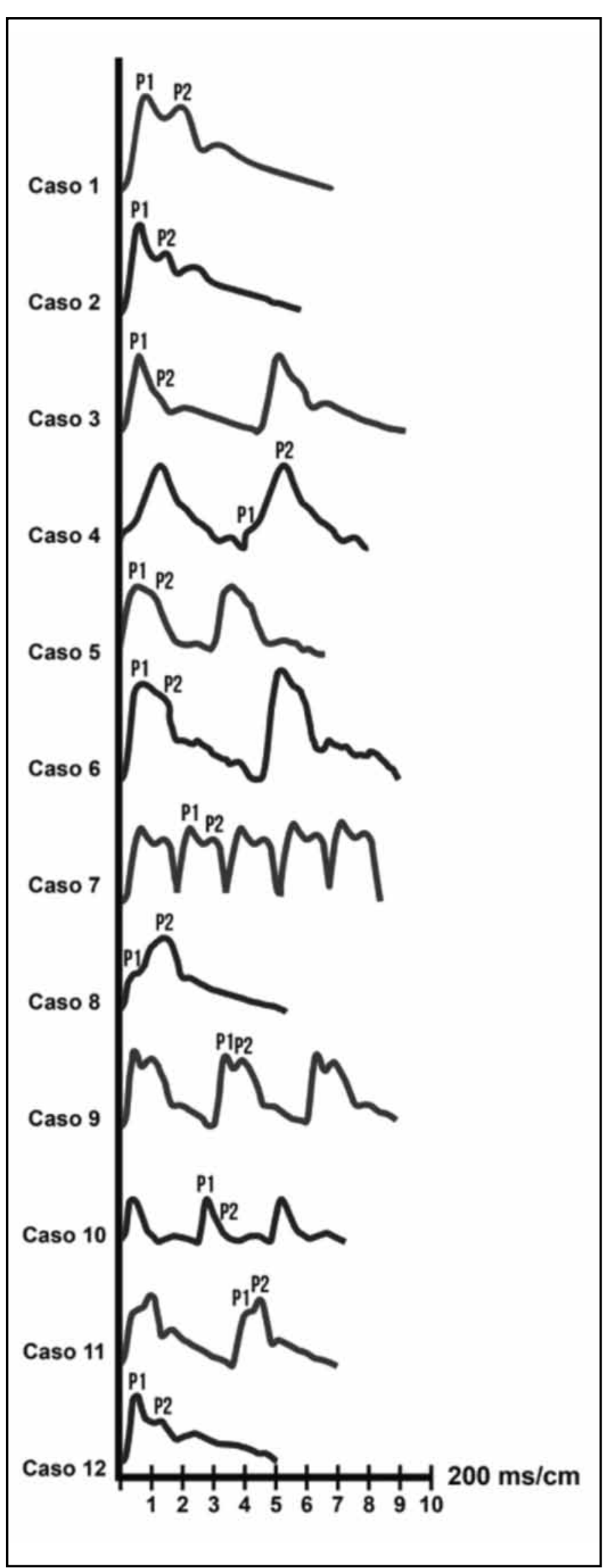

Figura 1. Casos. Trazos de onda HTA.

Caso 1. Trazo de onda de pulso arterial normal: $P 1$ mayor que $P 2$, separadas adecuadamente y la amplitud de pulso $(A P=P 2-P 1)$ negativa. Nótese que la PASC es similar a la $P A S$, la VOP es menor de $9 \mathrm{~m} / \mathrm{seg}$ y es menor al promedio de individuos con la misma edad, lo que se refleja en la edad-vascular menor a la real, el Aix-Braquial es negativo y el central es menor de $50 \%$, lo cual indica que estos parámetros se encuentran entre los rangos de la normalidad.

Caso 2. Trazo de onda de pulso arterial normal, P1 mayor que P2, separadas adecuadamente y AP es negativo, pero menor que el Caso 1, lo que refleja un Aix-Braquial más negativo y el central menor que el anterior. La VOP es normal, menor que el percentil 50 (23-24) para la edad y menor que el caso 1, evidenciando los efectos benéficos del ejercicio aeróbico. Lo cual se refleja también en la edad-vascular.

Caso 3. Trazo de onda de pulso arterial normal, P1 mayor que P2, separadas con menor distancia que los anteriores, pero se mantiene el AP negativo. La VOP está elevada para la edad del paciente, lo cual sugiere inicios de rigidez vascular, aumento de riesgo cardiovascular, se debe interpretar a la luz de la clínica por la presencia de factores de riesgo.

Caso 4. Trazo de onda de pulso arterial anormal, Pl es menor que P2 y el AP es positivo, lo cual indica que hay rigidez marcada, P2 es mayor y está elevando la presión sistólica, de igual manera se evidencia que la presión central es mayor que periférica, otro marcador de rigidez, lo cual se correlaciona con la VOP elevada. Se puede entender el componente mecánico de la endoprótesis en el sistema arterial con poca distensibilidad (compliance) (Figura 2).

Caso 5. Trazo de onda de pulso arterial anormal para la edad, P1 mayor que P2, pero no separadas adecuadamente y AP negativo. Teniendo en cuenta la edad de la paciente, se ven cambios en la onda, evidenciando una marcada rigidez. De igual manera, la VOP está muy aumentada para esta paciente, correlacionando los efectos de la hipercolesterolemia en la pared de los vasos, y el resultado crónico es la rigidez arterial.

Caso 6. Trazo de onda de pulso arterial anormal para la edad, $P 1$ es mayor que P2, pero no separadas adecuadamente y AP negativo. Se evidencian que ya hay cambios crónicos; la presión central es mayor que la presión periférica y la VOP está aumentada.

Caso 7. Trazo de onda de pulso arterial normal para la edad, P1 es mayor que P2 y AP es negativo. Se evidencia que, a pesar de los antecedentes del paciente, el control y buen manejo controla la progresión de la enfermedad. Posiblemente la rigidez arterial de este paciente antes era mayor, pero ha seguido un manejo estricto.

Caso 8. Trazo de onda de pulso arterial anormal, P1 menor que la $P 2$ y AP positivo. Los índices y la VOP son severamente altos, se evidencia la correlación entre las condiciones del paciente y la rigidez arterial. La presión arterial central es mayor que la periférica, lo cual demuestra que ya hay cambios crónicos y se correlacionan con los otros parámetros.

Caso 9. Trazo de onda de pulso arterial normal para la edad, P1 mayor que P2 y AP negativo. Se evidencia que las presiones están elevadas y la presión central es igual a la periférica, evidenciando cambios crónicos, al igual que una VOP elevada. Se demuestra que las enfermedades del paciente con un mal manejo se asocian a una rigidez arterial marcada para la edad del paciente.

Caso 10. Trazo de onda de pulso arterial anormal para la edad, P1 mayor que P2 y AP negativo. Se evidencia que las presiones están elevadas a pesar de un manejo agresivo, la presión central es igualmente alta pero menor a la periférica, lo cual evidencia que no hay cambios crónicos hasta el momento. La VOP es elevada para la edad. Por el contrario, se evidencian unos índices de aumentación muy bajos, lo cual puede estar relacionado al consumo de los medicamentos mencionados. evidenciando de cierta manera que el paciente tiene un adecuado manejo a pesar de tener las presiones elevadas en el momento de la medición.

Caso 11. Trazo de onda de pulso arterial anormal para la edad, P2 mayor que P1 y AP positivo. Se evidencia que las presiones están muy elevadas para la edad, la presión central es mayor que la periférica, lo cual evidencia cambios marcados. debido a las características del sistema circulatorio. La VOP es muy elevada para la edad al igual que los índices de aumentación. Debido a las características anatómicas (coartación severa) de la paciente se explica su rigidez arterial severa y la inversión de los picos de la onda de presión (Figura 3).

Caso 12. Trazo de onda de pulso arterial normal, P1 mayor que P2 y AP negativo. Se evidencia que las presiones están elevadas para la edad, pero en comparación con el caso anterior son significativamente menores, la presión central es menor a la periférica. $L a$ VOP es elevada para la edad, pero dentro de parámetros normales, de igual manera los índices de aumentación son menores (Figura 4). 


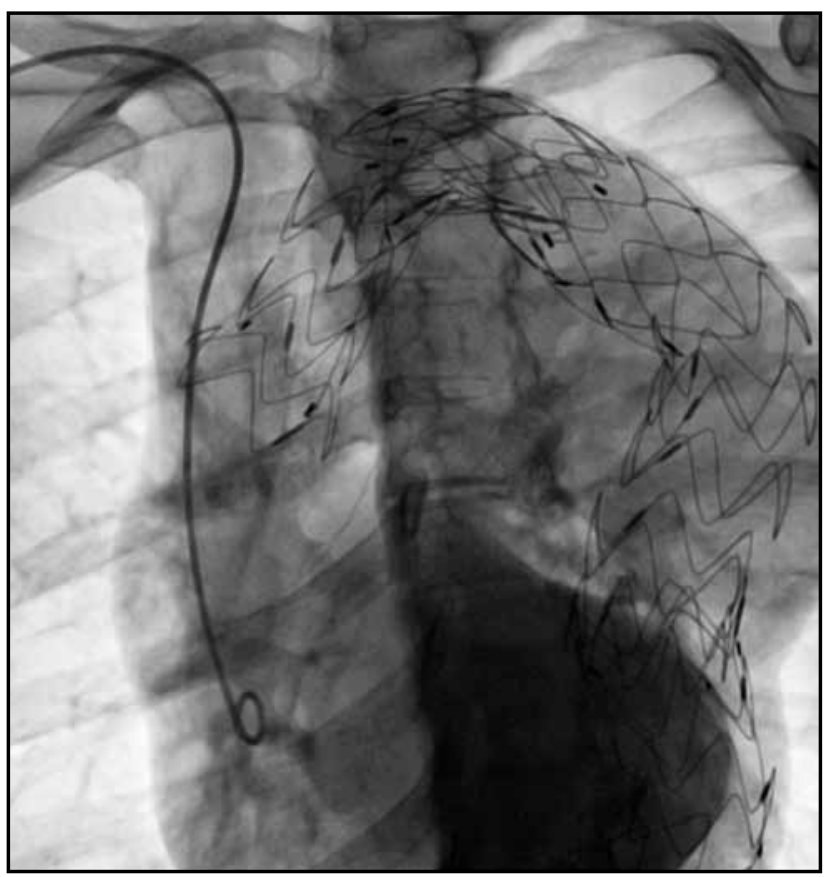

Figura 2. Aortograma: válvula aórtica trivalva, con insuficiencia aórtica grado III, ventrículo izquierdo con hipoquinesia generalizada, FEVI del $45 \%$, prótesis endovascular desde la aorta ascendente hasta aorta toracoabdominal.

confiables y no-invasivos) y debería utilizarse en prevención primaria y secundaria. A continuación, se describen doce casos relevantes de nuestra práctica real, para mostrar a la comunidad científica la importancia de estas mediciones, la interpretación y la asociación que hay con diferentes patologías.

\section{Presentación de casos}

En la Tabla 1 se muestran las principales características, manejo farmacológico y los parámetros de función vascular de los doce casos que se presentan. Asimismo, en la Figura 1, se ilustran las ondas de presión de pulso arterial de cada caso (Caso 1-12), haciendo énfasis en el pico sistólico (P1) y el pico de reflexión de la onda (P2).

El cuarto caso, es una paciente de 68 años con historia clínica de disección de aorta de10 años de evolución y dos correcciones con endoprótesis (Figura 2).

Por último, se presentan dos casos de niños con coartación de aorta. La primera de 12 años, recién diagnosticada sin corrección (Figura 3) en manejo médico, y el último, es un niño de 12 años en su segundo día de corrección con stent no-cubierto (Figura 4).

\section{Discusión}

Teniendo en cuenta los casos descritos, se tiene un concepto de rigidez arterial muy interesante en el que se puede ver como varía la VOP, presión periférica/central, edad-vascular y Aix, según ciertas patologías o características de una persona. Además, estos casos sirven para entender aún más el comportamiento y los mecanismos fisiopatológicos de la

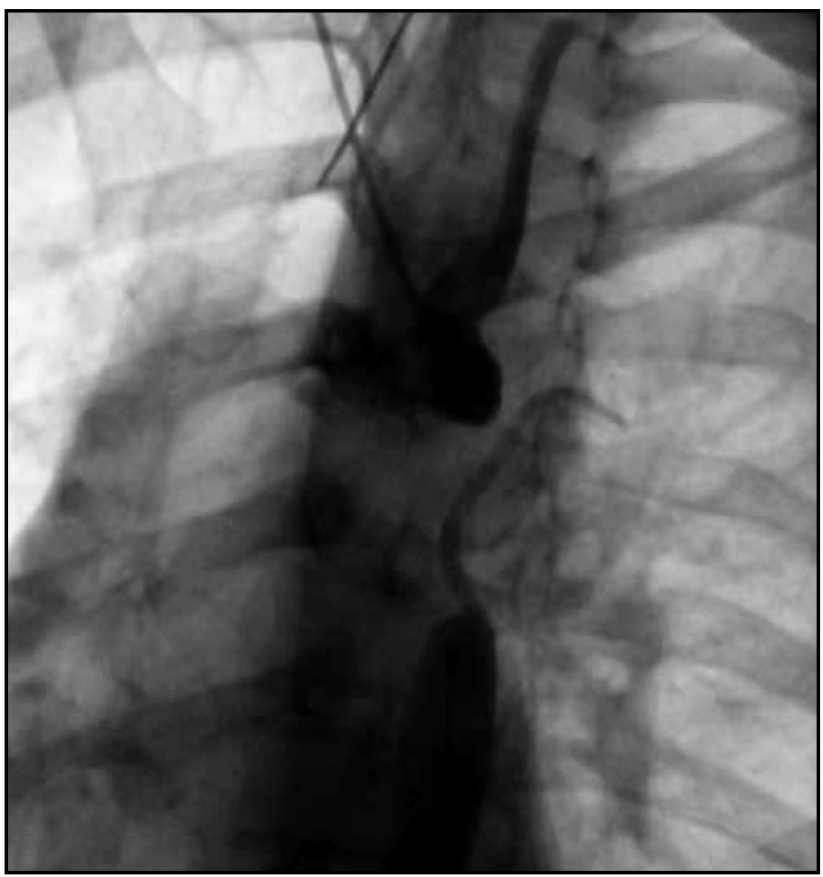

Figura 3. Aortograma: Interrupción del arco aórtico con segmento desconectado de cerca de $4 \mathrm{~cm}$, desde la aorta transversa a la aorta descendente, con compromiso de la subclavia izquierda que se encuentra ocluida. Colaterales arterio-arteriales muy abundantes.

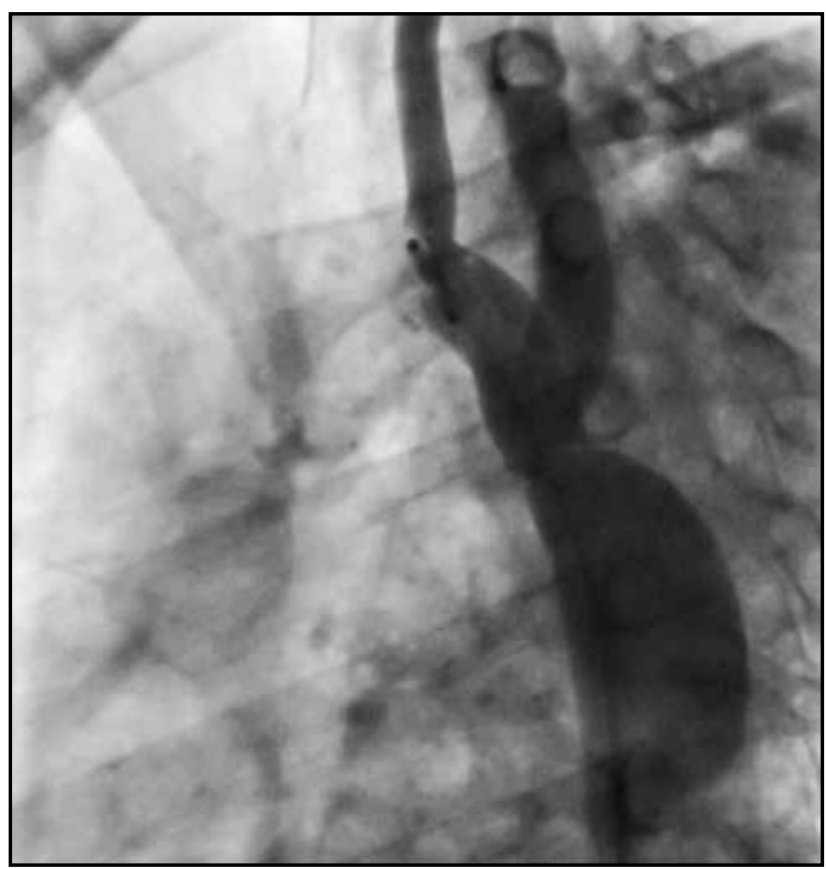

Figura 4. Aortograma: Se evidencia la corrección de la coartación. A pesar de no tener el mismo diámetro de la aorta descendente, el diámetro es mayor y hay un menor gradiente.

rigidez arterial en el sistema cardiovascular. A la luz de los conocimientos actuales y las herramientas que se disponen, es evidente que el examen físico y la simple toma de presión arterial braquial en el consultorio, no reflejan la salud vascular de un paciente. 
En primer lugar, se observa como el ejercicio aeróbico puede ser un factor protector y como los factores de riesgo (cigarrillo, obesidad, sedentarismo, hipertensión arterial, hiperlipidemia, diabetes y anomalías congénitas) pueden aumentar la VOP, incluso en quienes tienen la presión arterial periférica normal. De esta manera, se encuentra la VOP como uno de los marcadores tempranos de riesgo cardiovascular, resaltando la importancia de la VOP en la prevención primaria y secundaria.

De igual forma, hay cambios en la VOP en pacientes con patología mecánica de la aorta, como son los casos de disección de aorta corregida con endoprótesis o coartación de aorta, a pesar de ser, patologías y pacientes diferentes, sus componentes mecánicos se comportan de igual manera (menos distensibilidad arterial). En el caso de la corrección con endoprótesis, se observan los parámetros severamente elevados, la VOP y los índices evidencian una rigidez severa, la presión arterial central es mayor que la presión arterial periférica. Estos efectos pueden ser causados por las condiciones clínicas de la paciente y la endoprótesis persé. La endoprótesis es de un material menos elástico que la pared vascular y en este caso cubre la aorta ascendente y torácica (región con mayor distensibilidad), lo cual puede correlacionarse con la severidad de la rigidez. Este concepto puede estar presente en los casos de coartación aórtica, en donde la aorta a pesar de ser "joven y elástica", en una porción hay una gran estrechez, lo cual compromete la elasticidad y comportamiento fisiológico normal, por esta razón se puede ver como los parámetros están aumentados, las presiones, VOP y los índices son severamente elevados para la edad. En el caso del paciente después de la corrección con stent, se observa como mejoran los parámetros significativamente.

En los dos casos con dislipidemia se presentan dos extremos, en el caso de hiperlipidemia familiar homocigota, donde a pesar de tener controlados sus niveles de lípidos y trasplante de hígado, ya posee complicaciones vasculares en una edad temprana, lo cual se correlaciona de igual manera con los parámetros de rigidez arterial. En cambio, el otro caso es una paciente con dislipidemia no controlada, que igualmente muestra cambios, pero en menor compromiso (VOP y presión central elevadas).

En los casos de hipertensión arterial se observan diferentes efectos hemodinámicos; los pacientes que tienen la patología controlada y en manejo médico optimo a pesar de tener comorbilidades, los parámetros de rigidez están controlados. En cambio, los pacientes con la enfermedad no controlada, los parámetros se encuentran elevados. Así, se evidencia la importancia de controlar estrictamente esta enfermedad (crónica y silenciosa), y que los parámetros permiten evaluar el adecuado tratamiento.

Asimismo, se evidencia como la VOP puede ser el único marcador de riesgo, cuando los otros parámetros son normales (presiones e índices), es importante resaltar que la inversión de las presiones (central > periférica) es un marcador de riesgo y demuestra que ya hay cambios crónicos en el sistema vascular, de igual forma lo hace el AP y los índices de aumentación, los cuales evalúan también el estado arterial periférico (9).

La cuantificación de la rigidez arterial, es una forma de valorar la salud cardiovascular. El incremento de la rigidez está usualmente relacionado con daño de la pared arterial, y ha sido ampliamente demostrado en diferentes condiciones, principalmente en hipertensos (5-7). Aunque, la relación entre la rigidez y eventos es continua, un umbral de $>12 \mathrm{~m} / \mathrm{seg}$, ha sido sugerido por las Directrices de la ESC-2007-ESH como lesión de órgano blanco en pacientes hipertensos de mediana edad (7), incluso un consenso de expertos reciente afirma que se debería ajustar a un umbral de $10 \mathrm{~m} / \mathrm{seg}$ (14). La VOP tiene recomendación II-B, en pacientes de riesgo intermedio y se han establecido valores de referencia en personas sanas y con factores de riesgo cardiovascular (19-20), destacando la importancia la práctica clínica, seguimiento y prevención cardiovascular.

\section{Referencias}

1. Cavalcante JL, Lima JA, Redheuil A, Al-Mallah MH. Aortic stiffness current understanding and future directions. J Am Coll Cardiol. 2011; 57 (14): 1511-22.

2. Townsend RR, Wilkinson IB, Schiffrin EL, Avolio AP, Chirinos JA, Cockcroft JR, et al. American Heart Association Council on Hypertension. Recommendations for improving and standardizing vascular research on arterial stiffness: A Scientific Statement From the American Heart Association. Hypertension. 2015; 66 (3): 698-722.

3. AlGhatrif M, Strait JB, Morrell CH, Canepa M, Wright J, Elango P, et al Longitudinal trajectories of arterial stiffness and the role of blood pressure: The Baltimore Longitudinal Study of Aging. Hypertension. 2013; 62: 934-41.

4. Willum-Hansen T, Staessen JA, Torp-Pedersen C, Rasmussen S, Thijs L, Ibsen $\mathbf{H}$, Jeppesen J. Prognostic value of aortic pulse wave velocity as index of arterial stiffness the general population. Circulation. 2006; 113: 664-70.

5. Vlachopoulos C, Aznaouridis K, Stefanadis C. Prediction of cardiovascular events and all-cause mortality with arterial stiffness: a systematic review and meta-analysis. J Am Coll Cardiol. 2010; 55 (13): 1318-27.

6. Cruickshank K, Riste L, Anderson SG, Wright JS, Dunn G, Gosling RG. Aortic pulse-wave velocity and its relationship to mortality in diabetes and glucose intolerance: an integrated index of vascular function? Circulation. 2002;106: 2085-90.

7. Mancia G, De Backer G, Dominiczak A, Cifkova R, Fagard R, Germano G, et al. Management of Arterial Hypertension of the European Society of Hypertension; European Society of Cardiology. Guidelines 2007; Guidelines for the Management of Arterial Hipertensión. The Task Force for the Management of Arterial Hyper-tension of the European Society of Hypertension (ESH) and of the European Society of Cardiology (ESC). J Hypertens. 2007; 25:1105-87

8. Blacher J, Asmar R, Djane S, London GM, Safar ME. Aortic pulse wave velocity as a marker of cardiovascular risk in hypertensive patients. Hypertension. 1999; 33: 1111-17.

9. Ben-Shlomo Y, Spears M, Boustred C, May M, Anderson SG, Benjamin EJ, et al. Aortic pulse wave velocity improves cardiovascular event prediction: an individual participant meta-analysis of prospective observational data from 17,635 subjects. J Am Coll Cardiol. 2014; 63: 636-46.

10. Vlachopoulos C, Xaplanteris P,Aboyans V, Brodmann M, Cífková R, Cosentino $\mathbf{F}$, et al. The role of vascular biomarkers for primary and secondary prevention. A position paper from the European Society of Cardiology Working Group on peripheral circulation: Endorsed by the Association for Research into Arterial Structure and Physiology (ARTERY) Society. Atherosclerosis. 2015; 241 (2): 507-32.

11. Stefanadis C, Dernellis J, Tsiamis E, Stratos C, Diamantopoulos L, Michaelides A, Toutouzas P. Aortic stiffness as a risk factor for recurrent acute coronary events in patients with ischaemic heart disease. Eur Heart J. 2000; 21: 390-6.

12. Safar ME, Blacher J, Jankowski P. Arterial Stiffness, Pulse Pressure, and Cardiovascular Disease-Is it Possible to Break the Vicious Circle? Atherosclerosis. 2011; 218: 263-71.

13. McEniery CM, Yasmin, Hall IR, Qasem A, Wilkinson IB, Cockcroft JR, ACCT Investigators. Normal Vascular Aging: Differential Effects on Wave Reflection 
and Aortic Pulse Wave Velocity The Anglo-Cardiff Collaborative Trial (ACCT). J Am Coll Cardiol. 2015; 46 (9): 1753-60.

14. Laurent S, Cockcroft J, Van Bortel L. Expert consensus document on arterial stiffness: methodological issues and clinical applications. Eur Heart J. 2006; 27: 2588-605.

15. O'Rourke MF, Staessen JA, Vlachopoulos C, Duprez D, Plante GE. Clinical applications of arterial stiffness; definitions and reference values. Am J Hypertens. 2002; 15: 426-44.

16. O'Rourke MF, Staessen JA, Vlachopoulos C, Duprez D, Plante GE. Clinical applications of arterial stiffness; definitions and reference values. Am J Hypertens. 2002; 15: 426-44.

17. Kadoglou NP, Moulakakis KG, Papadakis I, Ikonomidis I, Alepaki M, Lekakis J, Liapis CD. Changes in aortic pulse wave velocity of patients undergoing endovas- cular repair of abdominal aortic aneurysms. J Endovasc Ther. 2012; 19 (5): 661-6.

18. Lantelme P, Dzudie A, Milon H, Bricca G, Legedz L, Chevalier JM, Feugier P. Effect of abdominal aortic grafts on aortic stiffness and central hemodynamics. J Hypertens. 2009; 27 (6): 1268-76.

19. The Reference Values for Arterial Stiffness' Collaboration. Determinants of pulse wave velocity in healthy people and in the presence of cardiovascular risk factors: establishing normal and reference values. Eur Heart J. 2010; 31: 2338-50.

20. Van Bortel LM, Laurent S, Boutouyrie P, Chowienczyk P, Cruickshank JK, De Backer T, et al. Artery Society; European Society of Hypertension Working Group on Vascular Structure and Function; European Network for Noninvasive Investigation of Large Arteries. Expert Consensus Document on the Measurement of Aortic Stiffness in Daily Practice using Carotid Femoral Pulse Wave Velocity. J Hypertens. 2012: 30: 445-8. 